\title{
Anxiety Reduction and Emotional Responding After a Session of Yoga
}

\author{
Rodney K Dishman ${ }^{1 *}$, Derek C Monroe ${ }^{2}$ and Jianchun Yin ${ }^{3}$ \\ ${ }^{1}$ Department of Kinesiology, University of Georgia, USA \\ ${ }^{2}$ Department of Neurology, University of California-Irvine, USA \\ ${ }^{3}$ College of Physical Education, Shanghai Normal University, China
}

*Corresponding author: Rodney K Dishman, Department of Kinesiology, University of Georgia, USA.

Received Date: November 15, 2019

Published Date: November 27, 2019

\begin{abstract}
Background: Anxiety reductions after traditional forms of exercise are typically small and most likely to occur after high intensities. Whether yoga, an alternative mindful exercise that is commonly practiced at light-to-moderate intensities, reduces anxiety is poorly understood.

Methods: Here, we examined whether 20 minutes of self-directed Hatha Yoga practice would protect against elevated anxiety and alter emotional processing measured as valence-modification of the acoustic startle eye blink response during emotional picture viewing.

Results: Compared to equally timed sessions of leg cycling exercise at self-selected intensity and quiet rest, yoga practice mitigated the increase in state anxiety elicited by picture viewing and subsequently protected against elevated anxiety during a second exposure to the emotional scenes. These effects were large enough (about one-half standard deviation) that they could be clinically meaningful. Yoga also attenuated the magnitude of the acoustic startle response to both pleasant and unpleasant picture content, but anxiety reduction after yoga was independent of the changes in startle response.
\end{abstract}

Conclusion: The results extend past findings by showing that yoga practice mollifies anxiety elicited by emotionally arousing pictures without altering emotional processing.

Keywords: Acoustic startle reflex; Affect; Exercise; Mind body therapies; Psychophysiology

\section{Introduction}

Randomized trials show that regular exercise is accompanied by clinically relevant reductions in symptoms of anxiety [1,2], and experimental studies of a single session of exercise show a small reduction in state anxiety that varies according to the type and intensity of the exercise and the comparator used. Reductions in state anxiety have been most consistent after treadmill exercise at high intensities when compared to quiet rest [3]. However, with the exception of one study of yoga practice, the types of exercise in those published reports were limited to a prescribed exposure to traditional forms including walking or running on a treadmill, leg cycling, stair climbing, or resistance exercise rather than a mode of exercise routinely practiced by participants prior to their acute exposure in a laboratory or clinic.
Yoga, an alternative mindful exercise that is commonly performed at light-to-moderate intensities [4,5], is practiced by an estimated $10 \%$ of U.S. adults [6]. It has shown some promise as an adjuvant for treating anxiety and its comorbidities of depression, sleep disorders, and schizophrenia [7-10]. However, we could find only three experimental studies of state anxiety after a session of yoga practice [11-13]. Just one of those studies compared yoga to traditional exercise. In a sample of 40 psychiatric inpatients diagnosed with schizophrenia, anxiety was reduced after 30 minutes of Hatha Yoga or 20 minutes of leg cycling exercise at self-selected intensity when each condition was compared to 20 minutes of quiet rest or reading [13]. 
Here, we studied a sample of yoga practitioners who did not have a known psychiatric disorder. Although they did not have elevated anxiety, we expanded on past research [3] by eliciting an emotional response in these participants prior to, and again after, exercise to test whether a change in emotional processing after yoga practice might help explain its expected anxiolytic effect. We also examined whether yoga might further protect against elevated anxiety in response to subsequent emotional provocation, as reported in a recent study of cycling exercise [14]. Emotional processing was assessed by affective ratings of emotional pictures and objectively by valence-modification of the acoustic startle eye blink response during picture viewing, which was not affected by leg cycling in a prior report [15].

Contemporary theorists characterize emotions as being organized along orthogonal dimensions of affective valence (i.e., pleasantness) and affective arousal. The two primary motivational systems along the valence dimension are the appetitive system, governing approach toward pleasant stimuli, and the defensive system, governing withdrawal from unpleasant, averse stimuli $[16,17]$. Emotional priming of these systems can be evidenced by the magnitude of the acoustic startle eye blink response (electromyographic measurement of the obligatory eye blink reflex after an acoustic startle probe), which is potentiated when processing unpleasant foreground stimuli (e.g., faces or scenes) but inhibited when processing pleasant stimuli [17]. An augmented startle response is seen in several anxiety disorders [18] and when anxiety is experimentally induced in people without an anxiety disorder [19]. Administration of anxiety-reducing drugs (e.g., benzodiazepines) to healthy people results in decreased startle response magnitude to unpleasant or threatening stimuli [20-22].

The aim of the study we report here was to examine whether yoga practice elicits a reduction in anxiety after emotional priming by exposure to scenes that elicit defensive or appetitive emotional systems. We compared responses after yoga practice, leg cycling exercise at self-selected intensity, and quiet rest. We hypothesized that state anxiety and the magnitude of the acoustic startle eye blink response during affective picture viewing would be attenuated after yoga practice but not after leg cycling exercise or quiet rest. We also hypothesized that decreased state anxiety would be positively associated with decreased startle magnitude.

\section{Materials and Methods}

\section{Recruitment}

Protocols complied with the Declaration of Helsinki and were approved by the Institutional Review Board. Students were contacted via their university e-mail address and were invited to complete an online screening questionnaire (www.qualtrics.com). Individuals 18-35 years old who reported at least 150 minutes of moderate-to-vigorous physical activity in the week prior to screening, experience with yoga (at least once a month for 6 months or more), and the ability to perform 20 minutes of selfdirected yoga were invited to participate in the study. Individuals with more than one risk factor for cardiovascular disease, a history of cold sensitivity (e.g. Reynaud's syndrome), who were seeking clinical treatment for psychological disorders, or currently taking psychoactive drugs were ineligible for the study.

\section{Participants}

A total of 229 undergraduates attending the University of Georgia responded and were screened. Of those, 45 were eligible and 23 were enrolled (i.e. gave informed consent to participate in the experiment and participated in at least one of the three visits). Six participants dropped out of the study for various reasons, and two participants completed the three visits but did not have useable eye blink data at one or more sessions. Here we report on 11 females and 4 males who had regularly practiced Hatha Yoga for six months to 12 years and completed the experimental protocol. They were aged 20 to 35 years $(26.1 \pm 5.2)$, moderately active, reporting 26 to 377 minutes per day of moderate, hard, or very hard physical activity the past week (133 \pm 96$)$, and most had normal weight with a body mass index (kg weight/m2 height) of 19.5 to 37 (23.8 \pm 4.3 ).

\section{Procedures}

The seven-day physical activity recall validated for college students [23] was used to measure moderate-to-vigorous leisure time physical activity level the week prior to the study. Participants complete a medical questionnaire to confirm they were healthy enough to complete 20 minutes of moderate-intensity cycling exercise. Participants also reported their experience with yoga and their medication use. Participants completed 3 laboratory visits scheduled at least 2 days apart. On their first visit to the laboratory, participants provided informed consent and screening for contraindications to exercise was accomplished with the Physical Activity Readiness Questionnaire [24]. Participants completed each of three randomly assigned conditions: a self-directed yoga session, a leg cycling session on a stationary cycle at self-selected intensity, and a quiet rest condition when the participant sat quietly in a chair in the cycling exercise room. Cycling was done on a Lode electronicallybraked cycle ergometer.

Warm-up (3 minutes, 25 Watts) was followed by 20 minutes of cycling at a self-selected intensity. Participants cycled at 40 Watts and were given the choice to maintain, increase, or reduce resistance by "a lot" (15 watts) or "a little" ( 5 watts) every five minutes until the end of the 20-minute bout ( 3 times total). Participants cooled down for 2 minutes at 25 Watts. There was a linear increase $(\mathrm{p}<.001)$ in power output from (mean \pm SD) $52.3 \pm 4.2$ watts to 68.3 \pm 11.8 watts. Average HR during the 20 -minute session was $60 \pm$ 7.7 watts. Heart rate was measured continuously during cycling exercise using a Polar ${ }^{\circledR}$ Vantage XL heart rate monitor (Polar Electro, Inc., Woodbury, NY, model 145900). There was a linear increase $(\mathrm{p}<.001)$ in HR from (mean \pm SD) $103 \pm 19$ bpm to 121 $\pm 26 \mathrm{bpm}$. Average HR during the 20 -minute session was $113 \pm 23$ bpm, $55 \% \pm 12 \%$ of age-estimated maximum HR, which is light-tomoderate intensity [25]. Yoga was completed in a secluded room. Participants were instructed to conduct a familiar warm-up for 3 minutes prior to, and 2 minutes of a familiar cool-down routine (savasana) after, 20 minutes of self-directed yoga poses (asanas). There was no change ( $p=.672)$ in HR from (mean \pm SD) $100 \pm 22$ bpm to $98 \pm 19 \mathrm{bpm}$. Average HR during the 20-minute session was 
$100 \pm 20 \mathrm{bpm}, 50 \% \pm 10 \%$ of age-estimated maximum HR. Heart rate during Hatha Yoga in practitioners typically ranges between 80 to $115 \mathrm{bpm}$, or about $50-55 \%$ of maximum HR $[4,5]$. Heart rate did not change during quiet rest $(\mathrm{p}=.414)$ and averaged $74 \pm 17 \mathrm{bpm}$.

\section{State anxiety}

Anxiety was assessed by the state version (X1) of the State-Trait Anxiety Inventory [26] administered at baseline after participant preparation, and again prior to and at the end of each affective picture show presentation.

\section{Affective pictures}

Three randomly assigned presentations of affective pictures were used to manipulate emotional state during each of the three conditions. Participants viewed each presentation on a screen approximately 21 inches from their face while seated in a semirecumbent position. Each affective presentation included 18 scenes; 6 unpleasant, 6 pleasant, and 6 neutral photos chosen from the International Affective Picture System (IAPS) image pool [27]. All pleasant and pleasant photos were rated as arousing [28], and each photo was only viewed once throughout the experiment [29]. Each IAPS photo appeared for 6 seconds. During the 10 seconds after each slide was presented, the participants provided affective ratings of valence and arousal using the Self-Assessment Manikin (SAM) rating scale. Ratings were followed by a random intercluster interval ranging between 3-5 seconds. Presentations lasted between 8 minutes and 55 seconds and 17 minutes and 35 seconds, depending on how long each participant took to respond to the SAM scales.

\section{Affective ratings}

The SAM rating scales [30] are analog scales used to measure subjective reports of affective valence and arousal. The SAM is a pictogram that exhibits levels of affective experience on independent continua of valence (from pleasant to unpleasant) and arousal (high arousal to low arousal). This is a 9-point scale that is marked with a pen or pencil. Participants were asked to rate their emotions felt during the presentation of each picture during the slide shows. Scores at averaged across each picture content (pleasant, neutral, unpleasant) at baseline before each condition were compared to published norms [27] for the slides employed to confirm that the slide content was perceived as intended.

\section{Acoustic startle probe}

The acoustic startle probe was administered once per picture during each affective presentation. The second and third pictures in each cluster (12 of 18 pictures in presentation) were accompanied by a startle probe administered randomly between 2.5 and 5.5 seconds following picture onset. The startle probe was a $50 \mathrm{~ms}$ burst of $95 \mathrm{~dB}$ (A) broadband white noise produced by a Coulbourn Instruments audio source module (model V85-05) and amplified by a RadioShack 40-watt amplifier. It was delivered binaurally through Sony model MDR-V200 dynamic stereo headphones, with an instantaneous rise/fall time [31]. The intensity of the acoustic stimulus was calibrated at the surface of the headphone using a sound level meter (General Radio Company, Concord Massachusetts).

\section{Electromyography (EMG)}

EMG recordings of the obicularis oculi provided recordings during each presentation of emotional scenes according to standard procedures [32]. The surface of the skin was lightly abraded, and two miniature biopotential skin electrodes filled with Mansfield R \& DTD-40 electrode gel were attached $5 \mathrm{~mm}$ lateral from the exocanthion and $10 \mathrm{~mm}$ medial and $5 \mathrm{~mm}$ inferior to that location. Electrode impedance was verified as less than $10 \mathrm{kohm}$ using a Grass electrode impedance meter (model EZM 4). EMG signals were amplified 100 times using a Grass P5 series AC amplifier, digitized at $1000 \mathrm{~Hz}$, and displayed using Spike2 version 6.08 software. Recorded data incl the mean amplitude and its standard deviation of the amplitude for the 1 second prior to probe onset, amplitude at probe onset, and peak amplitude within a response window of 20-100 milliseconds after probe onset. All amplitude measures were integrated, rectified, and expressed in millivolts. Response amplitude was designated as the difference between amplitude at probe onset and peak amplitude. Trials with excessive background noise and trials during which the subject was blinking at the time of probe onset were excluded.

Excessive background noise was defined as trials for which the standard deviation of the amplitude during the 1 second prior to probe onset was greater than or equal to $3 \mathrm{SD}$ from the mean standard deviation within each subject per visit. Spontaneous eye blinks at probe onset were identified as $\geq 3$ SD from the mean amplitude of the 1 second prior to probe onset for each trial. Finally, response amplitudes $<2$ SD from the amplitude at probe onset were considered non-responses. A total of 51 startle responses were excluded because of excessive noise or blinking and 118 trials were excluded as non-responses, leaving a total of 1451 valid startle responses out of 1620 probes (90\%). Response magnitude was calculated as the product of mean response amplitude in millivolts and the response probability $\left(\mathrm{mM}=\mathrm{mA}^{*} \mathrm{P}\right)$, where response probability represents the proportion of valid responses out of total possible responses for each valence [32]. Response magnitudes were transformed and expressed as z-scores within each participant for each day of testing to control for differences in basal EMG activity between subjects and within subjects across visits.

\section{Statistical analyses}

Statistical analyses were conducted using IBM SPSS version 22. A3 condition (yoga, cycling, rest) $\mathrm{x} 3$ valence (positive, neutral, negative) $\mathrm{x}$ time repeated measures ANOVA tested the hypothesis of condition $\mathrm{x}$ time effects on state anxiety and condition $\mathrm{x}$ time $x$ valence effects on emotional modulation of ASER magnitude. Interactions were decomposed using orthogonal polynomial contrasts for time and condition and Helmert contrasts of yoga with the cycling and rest conditions. Follow-up simple main effects of time within condition were also conducted by RM-ANOVAs within condition and valence. When assumptions of sphericity were 
violated, we reported epsilon and Huynh-Feldt corrected estimates. Effect sizes associated with the F statistics are expressed as $\eta^{2}$. Statistical power to detect a moderate effect $\left(\eta^{2} \geq .10\right)$ exceeded .80 at an alpha of .05 [33].

To determine whether startle eye blink magnitude was directly related to state anxiety, 3-Condition (yoga, cycling, quiet rest $\mathrm{x}$ 4-Time $\mathrm{x}$ Sex mixed model ANCOVAs with time-varying covariates were also conducted. Reduction in the F statistic and partial $\eta^{2}$ in the adjusted (i.e., conditional) model, compared to the unconditional model, was interpreted as the startle eye blink magnitude accounting for a significant percentage of the change in state anxiety, i.e., 1 - (adjusted $\eta^{2} /$ unconditional $\eta^{2}$ ) [34].

\section{Results}

\section{State anxiety}

There was a linear interaction between condition and time when the yoga condition was contrasted with cycling and quiet rest $\left[\mathrm{F}(1,14)=4.878, \mathrm{p}=.044, \eta^{2}=.258, \varepsilon=.692\right]$ displayed in (Figure 1). Anxiety was increased by the first exposure to emotional scenes in all conditions and remained elevated after cycling and quiet rest $\left[F(1,14)=5.328, p=.037, \eta^{2}=.276, \varepsilon=0.656\right]$. In contrast, a quartic effect for time in the yoga condition $\left[F(1,14)=5.639, p=.032, \eta^{2}=\right.$ $.287, \varepsilon=1.0]$ indicated that anxiety was reduced to pre-scene levels after yoga practice and remained lower after the second exposure to emotional scenes.

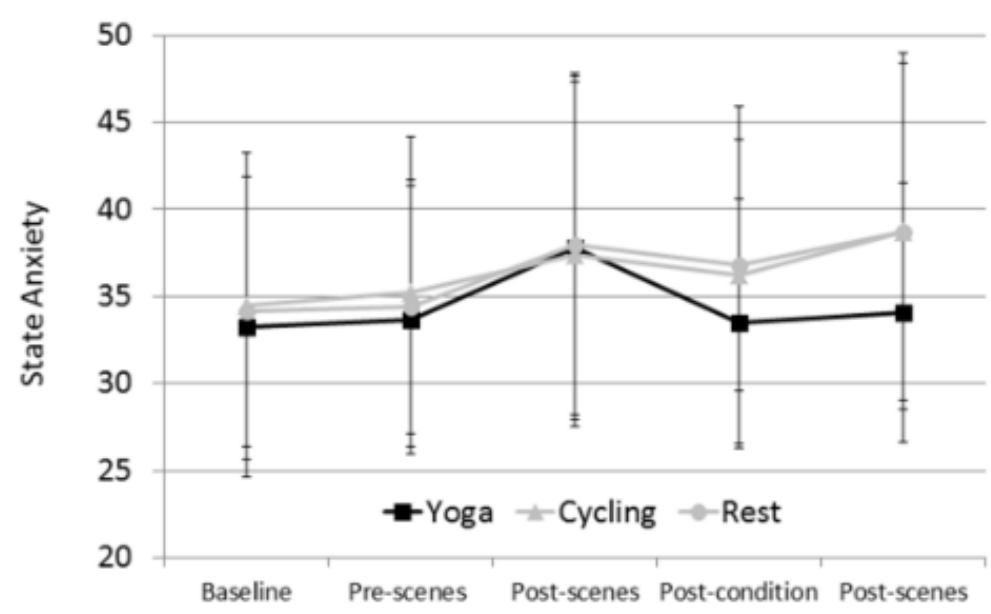

Figure 1: State anxiety responses to emotional scenes according to condition.

\section{Ratings of pleasantness and arousal}

There was a significant effect of scene valence on ratings of pleasantness $\left[\mathrm{F}(2,28)=298.396, \mathrm{p}<.001, \eta^{2}=.955, \varepsilon=0.912\right]$ and arousal $\left[F(2,28)=41.649, p<.001, \eta^{2}=.748, \varepsilon=0.725\right]$. See (Figure 2). Consistent with expected effects of picture content, pleasantness was higher for positive scenes $[F(1,14)=281.991, p$ $\left.<.001, \eta^{2}=.953\right]$, and lower for negative scenes $[\mathrm{F}(1,14)=407.515$, $\mathrm{p}<.001, \eta^{2}=.967$. Arousal was higher for negative scenes $[\mathrm{F}(1,14)$ $\left.=34.609, \mathrm{p}<.001, \eta^{2}=.712\right]$ but not for positive scenes $[\mathrm{F}(1,14)=$ 1.838, $\left.\mathrm{p}=.197, \eta^{2}=.116\right]$. The condition-by-time-by-valence effect was not significant for pleasantness $\left[\mathrm{F}(2,56)=1.777, \mathrm{p}=.108, \eta^{2}=\right.$ $.113, \varepsilon=0.805]$ or arousal $\left[\mathrm{F}(2,56)=0.799, \mathrm{p}=.597, \eta^{2}=.054, \varepsilon=\right.$ $0.931]$.
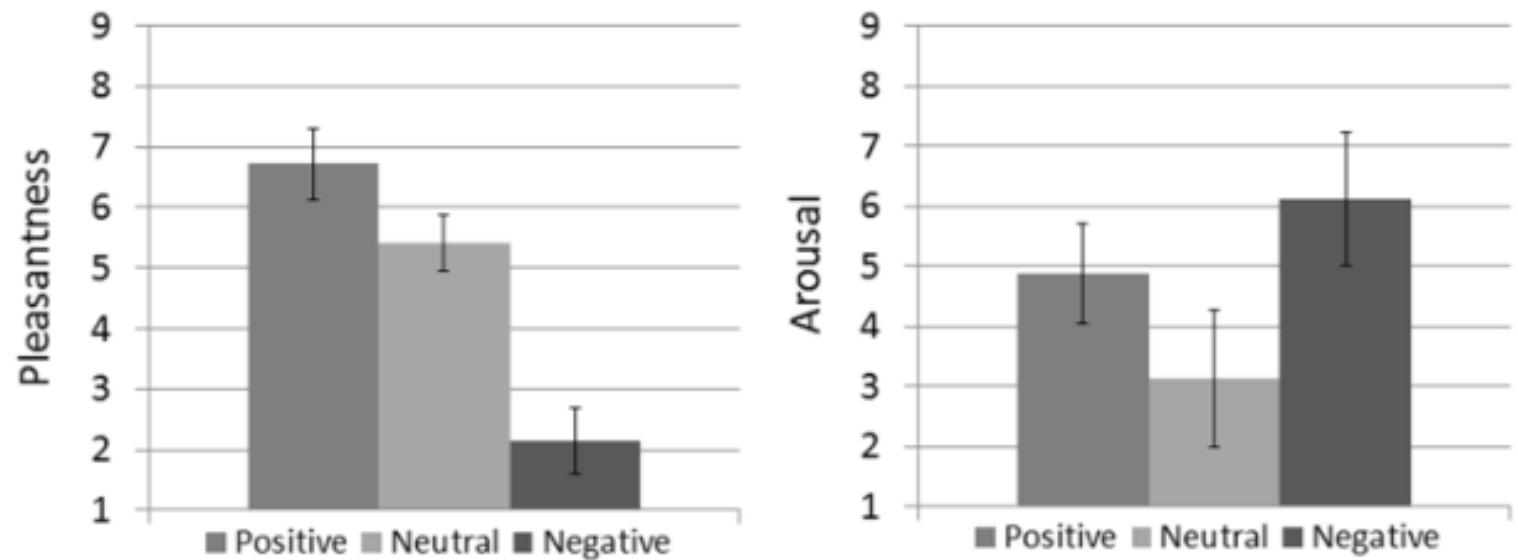

Figure 2: Affective ratings according to valence of emotional scenes. 


\section{Eye blink response magnitude}

There was a significant effect of valence $[F(2,28)=4.147, p$ $\left.=.026, \eta^{2}=.229, \varepsilon=1.00\right]$. Consistent with expected effects of picture content, the magnitude of eye blink response was higher for negative scenes $\left[F(1,14)=6.592, p=.022, \eta^{2}=.320\right]$ and lower for positive scenes $\left[F(1,14)=6.006, p=.028, \eta^{2}=.300\right]$, and. See (Figure 3).

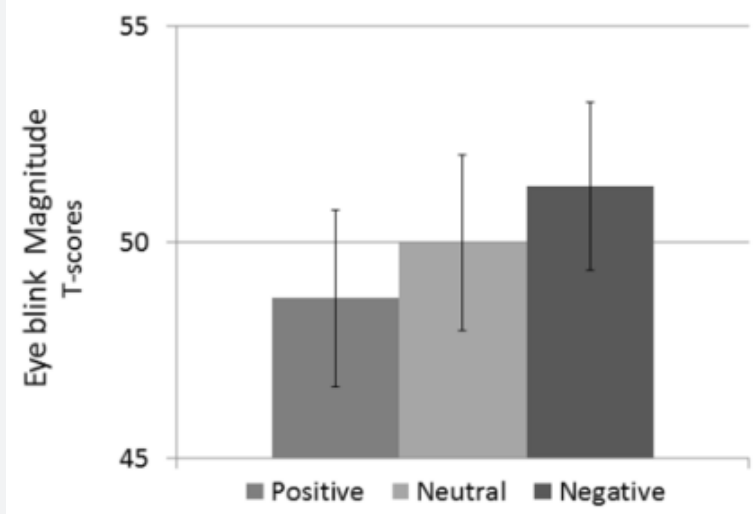

Figure 3: Acoustic startle eye blink responses according to valence of emotional scenes.

There was a 3-way linear interaction between condition, There was a linear decline in eye blink magnitude in response to time, and valence when the yoga condition was contrasted with unpleasant and pleasant scenes compared to neutral scenes, after cycling and quiet rest $\left[F(1,11)=5.969, p=.033, \eta^{2}=.352, \varepsilon=1.0\right] . \quad$ yoga compared to cycling and quiet rest. See (Figure 4$)$.

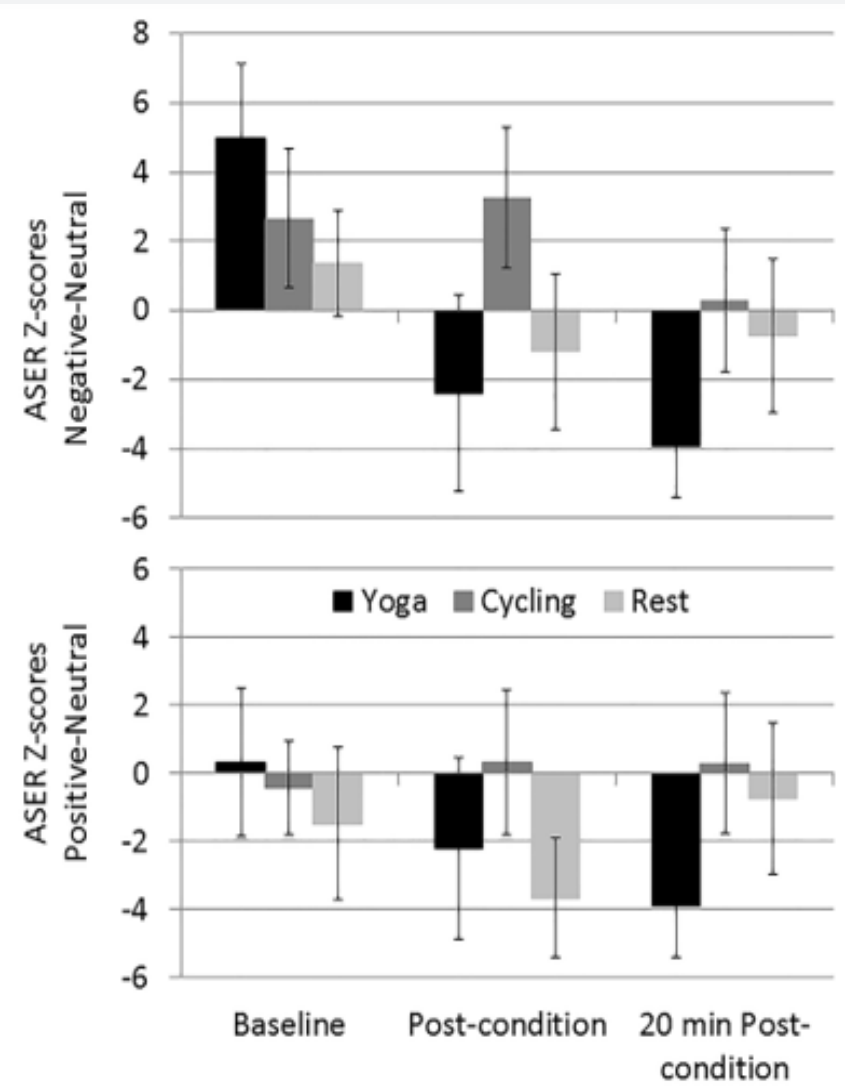

Figure 4: Acoustic startle eye blink responses according to condition by time for negative or unpleasant valence (top panel) and positive or pleasant valence (bottom panel) of emotional scenes.

Time-varying ANCOVA indicated that state anxiety after viewing pictures in the yoga practice condition, $\mathrm{F}(2,28)=3.62$, $\mathrm{p}=.040, \eta^{2}=.205$, was unrelated to eye blink magnitude in response to either unpleasant picture content (adjusted analysis: F $(2,27)$
$=3.69, \mathrm{p}=.038, \beta=.172, \mathrm{p}=.541$, and $\eta^{2}=.215$ ) or pleasant picture content (adjusted analysis: $F(2,27)=3.51, p=.044 \beta=.130, p=.645$, and $\left.\eta^{2}=.206\right)$, respectively. 


\section{Discussion}

The primary novel findings of the study are that a session of yoga practice mitigated the increase in state anxiety elicited by affective picture viewing and attenuated the magnitude of the acoustic startle response to both pleasant and unpleasant picture content, when compared to leg cycling exercise and quiet rest. The concurrent reductions in self-reported anxiety and the startle eye blink probe provide convergent subjective and objective evidence that yoga practice was anxiolytic. Yoga practice also protected against elevated state anxiety during a second exposure to the emotional scenes.

However, state anxiety after yoga was unrelated to startle eye blink response, and neither pleasantness nor emotional arousal in response to picture content was altered by yoga practice. Although yoga reduced anxiety after emotional priming by scenes that elicited either defensive- or appetitive-emotional systems, the hypothesis that anxiety reduction after yoga would be explainable by altered emotional processing was not supported. Neither yoga nor leg cycling altered the appraisal of picture content as pleasant, unpleasant or arousing. Thus, the results extend past findings by showing that yoga practice reduces anxiety elicited by emotionally arousing pictures and may provide anxiolytic prophylaxis against subsequent emotional provocation. However, like leg cycling exercise, yoga did not alter emotional processing measured here as valence-modification of the acoustic startle eye blink response during emotional picture viewing. Hence, we interpret the attenuated startle response after yoga as a general neural deactivation or relaxation effect [15].

The results agree with earlier reports from our laboratory that low- or moderate-intensity leg cycling exercise does not alter state anxiety or emotional responding measured by ratings of affective picture content and the acoustic startle eye blink response, when compared to quiet rest [15,35]. Here, participants chose to cycle at about $55 \%$ of maximum HR, similar to the average intensity of Hatha Yoga practice, which approximates an intensity that is less than $40 \%$ VO2peak, when given opportunities to change the intensity as they preferred. We did not assess whether participants viewed yoga practice or leg cycling as pleasant or unpleasant during each session, which might influence state anxiety after high intensity, but likely not the light intensity exercise used here [36]. We previously reported that moderate intensity leg cycling is evaluated as an emotionally neutral experience [37].

We are aware of only two other reports of randomized studies of anxiety after a single session of yoga practice. In a study of 86 men and women aged 18 to 64 years who had completed a onemonth yoga course, state anxiety was reduced in participants assigned to $20 \mathrm{~min}$ of shavasana (corpse pose) but not after 20 minutes of supine rest [11]. In a study of young male residents at a yoga center, anxiety was lowered more after about 10 minutes of cyclic meditation yoga postures followed by 10 minutes of corpse pose, when compared with an equal period of corpse pose only
[12]. Explanations for the anxiety reductions were not tested in those studies.

\section{Conclusion}

The results extend past findings by showing that Hatha Yoga practice reduced anxiety elicited by emotionally arousing pictures and provided prophylaxis against subsequent emotional provocation. The effects of Yoga were large enough (about one-half standard deviation) that they could be clinically meaningful. The reduction in the objective startle response concurrent with lowered self-reported anxiety supports that yoga practice is anxiolytic, but anxiety responses did not depend on the startle response, and like leg cycling exercise, yoga did not alter emotional processing of affective scenes.

\section{Acknowledgment}

None.

\section{Conflict of interest}

No conflict of interest.

\section{References}

1. Asmundson GJ, Fetzner MG, Deboer LB, Powers MB, Otto MW, et al. (2013) Let's get physical: a contemporary review of the anxiolytic effects of exercise for anxiety and its disorders. Depress Anxiety 30(4): 362373.

2. Herring MP, O Connor PJ, Dishman RK (2010) The effect of exercise training on anxiety symptoms among patients: a systematic review. Arch Intern Med 170(4): 321-331.

3. Ensari I, Greenlee TA, Motl RW, Petruzzello SJ (2015) Meta-analysis of acute exercise effects on state anxiety: an update of randomized controlled trials over the past 25 years. Depress Anxiety 32(8): 624-634.

4. Hagins M, Moore W, Rundle A (2007) Does practicing hatha yoga satisfy recommendations for intensity of physical activity which improves and maintains health and cardiovascular fitness? BMC Complement Altern Med 7: 40

5. Miles SC, Chun Chung C, Hsin Fu L, Hunter SD, Dhindsa M, et al. (2013) Arterial blood pressure and cardiovascular responses to yoga practice. Altern Ther Health Med 19(1): 38-45.

6. Clarke TC, Black LI, Stussman BJ, Barnes PM, Nahin RL (2015) Trends in the use of complementary health approaches among adults: United States, 2002-2012. Natl Health Stat Report (79): 1-16.

7. Balasubramaniam M, Telles S, Doraiswamy PM (2013) Yoga on our minds: a systematic review of yoga for neuropsychiatric disorders. Front Psychiatry 3: 117

8. Cabral P, Meyer HB, Ames D (2011) Effectiveness of yoga therapy as a complementary treatment for major psychiatric disorders: a metaanalysis. Prim Care Companion CNS Disord 13(4): PCC.10r01068.

9. Desveaux L, Lee A, Goldstein R, Brooks D (2015) Yoga in the Management of Chronic Disease: A Systematic Review and Meta-analysis. Med Care 53(7): 653-661.

10. Li AW, Goldsmith CA (2012) The effects of yoga on anxiety and stress. Altern Med Rev 17(1): 21-35.

11. Khemka SS, Rao NH, Nagarathna R (2009) Immediate effects of two relaxation techniques on healthy volunteers. Indian J Physiol Pharmacol 53(1): 67-72.

12. Subramanya P, Telles S (2009) Effect of two yoga-based relaxation techniques on memory scores and state anxiety. Biopsychosoc Med 3: 8.

13. Vancampfort D, De Hert M, Knapen J, Wampers M, Demunter H, et al. State anxiety, psychological stress and positive well-being responses to 
yoga and aerobic exercise in people with schizophrenia: a pilot study Disabil Rehabil 33(8): 684-689.

14. Smith JC (2013) Effects of emotional exposure on state anxiety after acute exercise. Med Sci Sports Exerc 45(2):372-378.

15. Smith CJ, O Connor PJ, Crabbe JB, Dishman RK (2002) Emotional responsiveness after low- and moderate-intensity exercise and seated rest. Med Sci Sports Exerc 34(7): 1158-1167.

16. Lang P J, Bradley MM, Cuthbert BN (1990) Emotion, attention, and the startle reflex. Psychol Rev 97(3): 377-395.

17. Lang PJ, Bradley MM, Cuthbert BN (1998) Emotion, motivation, and anxiety: brain mechanisms and psychophysiology. Biol Psychiatry 44(12): 1248-1263.

18. McTeague LM, Lang PJ (2012) The anxiety spectrum and the reflex physiology of defense: from circumscribed fear to broad distress. Depress Anxiety 29(4): 264-281.

19. Grillon C, Ameli R, Foot M, Davis M (1993) Fear-potentiated startle: Relationship to the level of state/trait anxiety in healthy subjects. Biological Psychiatry 33(8-9): 566-574.

20. Bitsios P, Philpott A, Langley, RW, Bradshaw, CM, Szabadi E (1999) Comparison of the effects of diazepam on the fear potentiated startle reflex and the fear-inhibited light reflex in man. J Psychopharmaco 113(3): 226-234.

21. Patrick CJ, Berthot BD, Moore JD (1996) Diazepam blocks fearpotentiated startle in humans. J Abnorm Psychol 105(1): 89-96.

22. Rodriguez Fornells A, Riba J, Gironell A, Kulisevsky J, Barbanoj, M] (1999) Effects of alprazolam on the acoustic startle response in humans. Psychopharmacology (Berl) 143(3): 280-285.

23. Dishman RK, Steinhardt M (1988) Reliability and concurrent validity for a 7-d re-call of physical activity in college students. Med Sci Sports Exerc 20(1): 14-25

24. Thomas S, Reading J, Shephard RJ (1992) Revision of the Physical Activity Readiness Questionnaire (PAR-Q). Can J Sports Sci 17(4): 338-345.

25. Armstrong L, Balady GJ, Berry MJ (2006) Exercise Testing. In Mitchel H. Whaley, Peter H Brubaker, Robert M Otto (eds.), ACSM's Guidelines for Exercise Testing and Prescription ( $7^{\text {th }}$ edn). Baltimore MD: American College of Sports Medicine Pp 36-126.
26. Spielberger CD, Gorsuch RL, Lushene R, Vagg PR, Jacobs GA (1983) Manual for the State-Trait Anxiety Inventory. Palo Alto, CA: Consulting Psychologists Press, USA.

27. Lang PJ, Bradley MM, Cuthbert BN (2008) International affective picture system (IAPS): Affective ratings of pictures and instruction manual. Gainesville, USA, Pp 1-61.

28. Cuthbert BN, Bradley, MM, Lang PJ (1996) Probing picture perception: Activation and emotion. Pscyhophysiology 33(2): 103-111.

29. Larson CL, Ruffalo D, Nietert JY, Davidson RJ (2000) Temporal stability of the emotion-modulated startle response. Psychophysiology 37(1): 92-101.

30. Bradley MM, Lang PJ (1994) Measuring emotion: The self-assessment manikin and the semantic differential. J Behav Ther Exper Psychol 25(1): 49-59.

31. Berg, WK, Balaban MT (1999) Startle elicitation: stimulus parameters, recording techniques, and quantification. In: Michael E Dawson, Anne M Schell, Andreas H Böhmelt (eds), Startle modification: implications for neuroscience, cognitive science, and clinical science. Cambridge, UK.

32. Blumenthal TD, Cuthbert BN, Filion DL (2005) Committee report: Guidelines for human startle eyeblink electromyographic studies. Psychophysiology 42(1): 1-15.

33. Faul F, Erdfelder E, Lang AG, Buchner A (2007) G*Power 3: a flexible statistical power analysis program for the social, behavioral, and biomedical sciences. Behav Res Methods 39(2): 175-191.

34. Winer BJ, Brown DR, Michels KM (1991) Statistical principles in experimental design, ( $3^{\text {rd }}$ edn). New York: McGraw-Hill, USA, pp 820 836.

35. Tieman JG, Peacock LJ, Cureton KJ, Dishman RK (2001) Acoustic startle eyeblink response after acute exercise. Int. J Neurosci 106(1-2): 21-33

36. Ekkekakis P, Parfitt G, Petruzzello SJ (2011) The pleasure and displeasure people feel when they exercise at different intensities: decennial update and progress towards a tripartite rationale for exercise intensity prescription. Sports Med 41(8): 641-671.

37. Crabbe JB, Smith CJ, Dishman RK (2007) Emotional and electroencephalographic responses during affective picture viewing after exercise. Physiol Behav 90(2-3): 394-404. 